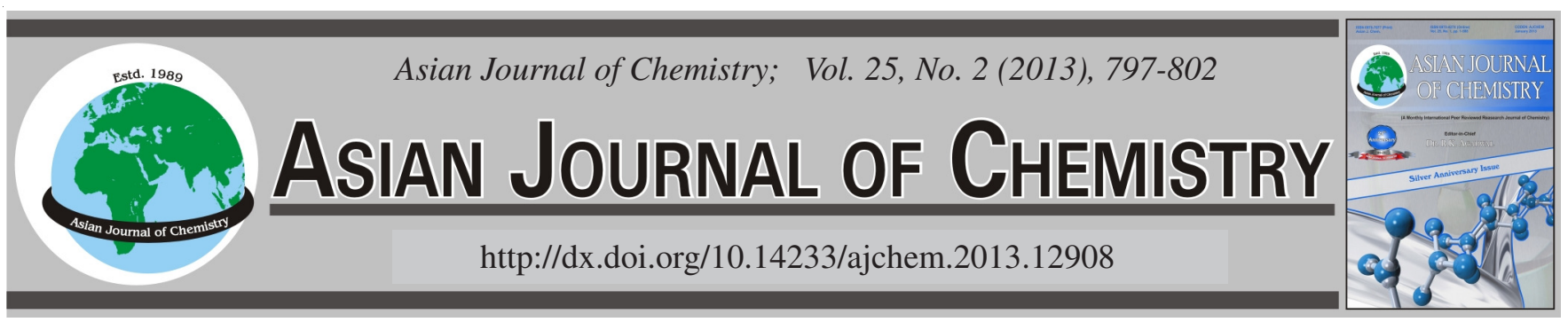

\title{
Comparative Enantioseparation of Crufomate with Cellulose- and Amylose-based Chiral Stationary Phases on Reverse-Phase and Normal-Phase High-Performance Liquid Chromatography
}

\author{
Tingting Chai ${ }^{1,2}$, Xueqing Wang ${ }^{1}$, Mei Bie ${ }^{1,2}$, Shouhui Dai ${ }^{2}$, Hualin Zhao ${ }^{2}$, Shuming Yang ${ }^{2}$ and Jing Qiu ${ }^{2, *}$
}

${ }^{1}$ Key Laboratory of Food and Biotechnology, Department of Bioengineering, School of Biotechnology and Food Science, Tianjin University of Commerce, Tianjin 300134, P.R. China

${ }^{2}$ Institute of Quality Standards and Testing Technology for Agro-Products, Key Laboratory of Agro-product Quality and Safety, Chinese Academy of Agricultural Sciences, Beijing 100081, P.R. China

*Corresponding author: Tel/Fax: +86 10 82106560; E-mail: jinn.qiu@ hotmail.com

\begin{abstract}
This study used cellulose- and amylose-based chiral stationary phases to comparatively separate crufomate enantiomers on reverse-phase and normal-phase high performance liquid chromatography (RP-HPLC and NP-HPLC). The baseline separations were obtained on Phenomene Lux cellulose-1, Lux cellulose-2 and Lux amylose-2 with 2-propanol/hexane and on Lux cellulose-1 and Lux amylose-2 with acetonitrile/water as mobile phase. The results of optical rotation detections showed that (+)-enantiomer of crufomate was firstly eluted on Lux cellulose-1 and Lux amylose-2 both on NP-HPLC and RP-HPLC, while the (-)-enantiomer was firstly eluted on Lux cellulose-2 and Lux cellulose- 3 on NP-HPLC. The influences of column temperature from $5-40{ }^{\circ} \mathrm{C}$ on separation were investigated on three chiral stationary phases. The thermodynamic parameters were calculated using van't Hoff equations to study the enthalpy-driven and entropy-driven process. This work will be helpful to prepare optical pure enantiomers and establish efficient analytical method for study on enantioselective behaviours of crufomate.
\end{abstract}

Key Words: Crufomate, Enantiomeric separation, HPLC, Elution order, Optical rotation.

\section{INTRODUCTION}

Since the first commercial organophosphorus pesticides (OPs) was produced in $1930 \mathrm{~s}^{1}$, organophosphorus pesticides are widely used as commercial chemicals in agriculture, for its high efficiency, high toxicity to pests and rapid degradation. It is predicted that organophosphorus pesticides have about $40 \%$ of the worldwide market and occupy an important position in the future ${ }^{2}$. With the wide recognition of the importance of chirality, stereogenic center was initially introduced into organophosphorus pesticides, in the late $1960 \mathrm{~s}^{3}$ and now $30 \%$ of organophosphorus pesticides are chiral. These chiral organophosphorus pesticides can be divided into three categories: chiral phosphorus centers, chiral carbon centers and chiral centers on both phosphorus and carbon atoms ${ }^{4}$. Several references report chiral organophosphorus pesticides have enantioselective biological activities, toxicology $y^{3}$ and different degradation rates ${ }^{5-8}$. Therefore, the pure enantiomeric forms are required by scientists for their bioactivity, toxicity and metabolic mechanisms. While racemates are mainly marketed and produced in the present ${ }^{9}$. The important access to obtain optical-enantiomer standards is separation of racemates with chromatography techniques, such as high-performance liquid chromatography (HPLC) and gas chromatography (GC) and capillary electrophoresis $(\mathrm{CE})^{10}$.

Crufomate (Fig. 1) is one of the most important organophosphorus pesticides. Crufomate is considered as an organic substitute for a highly toxic pesticide, used to handle livestock and prevent the torsaloes, parasites in vitro and intestinal worms. Its acute toxicity to rats have been studied, is only $1 / 10$ $1 / 20$ of methamidophos in oral way ${ }^{11}$. According to the chiral organophosphorus pesticides classes, crufomate contain only one asymmetric phosphorus center and correspondingly present two enantiomers.<smiles>CN[P+](=O)(OC)Oc1ccc(C(C)(C)C)cc1Cl</smiles>

Fig. 1. Chemical structure of crufomate 
The chromatographic techniques, including HPLC and capillary electrophoresis with a suitable chiral-selective columns and reagents, have been researched for resolution of crufomate enantiomers. HPLC with chiral stationary phases (CSPs) is one of the fastest growing methods. Ellington's team studied the enantioselectivity of crufomate on CHIRALCEL ${ }^{\circledR} \mathrm{AD}$, CHIRALCEL ${ }^{\circledR} \mathrm{OD}$ and CHIRALCEL ${ }^{\circledR} \mathrm{OJ}$ columns on normalphase HPLC (NP-HPLC) ${ }^{7}$. Some reports involved in enantioseparation of crufomate in the most common mode of capillary electrophoresis, named micellar electrokinetic chromatography (MECC or MEKC) with hydroxypropyl- $\beta$-cyclodextrin (HP$\beta$-CD), $\beta$-cyclodextrin $(\beta-C D)$ and/or $\gamma$-cyclodextrin $(\gamma-C D)^{12}$.

In present research, the enantioselectivity of crufomate on cellulose- and amylose-based chiral stationary phases on NP-HPLC and RP-HPLC were comparatively investigated. The influences of column temperature and the chiral stationary phases on resolution were also studied. The elution orders on different separation conditions were determined by an optical rotation $(\mathrm{OR})$ detector.

\section{EXPERIMENTAL}

Racemic standard and reagents: Racemic crufomate (95\%) was purchased from Dr. Ehrenstorfer GmbH (Germany). Stock solution was prepared by dissolving crufomate in 2-propanol (IPA). Working standard solution was prepared in acetonitrile (ACN)/water (50/50, v/v ) for RP-HPLC and the concentration was $100 \mathrm{mg} \mathrm{L}^{-1}$. For NP-HPLC, the working standard solution was prepared at $500 \mathrm{mg} \mathrm{L}^{-1}$ in hexane. ACN, IPA and hexane were HPLC grade (Fisher, UK). Water was prepared from a Millipore Milli-Q system.

Separations were achieved with an Agilent 1200 series HPLC equipped with G1322A degasser, G1311A quatpump, G1316B column compartment, G1315C diode array detector, G1329A autosampler and a $20-\mu \mathrm{L}$ sample loop (Wilmington, DE, USA). The UV signals were acquired and manipulated by an Agilent Chemstation. The optical rotation signals of crufomate enantiomers were identified by Chiralyser-MP optical rotation detector produced by Ibzmesstechnik Company (Germany) and provided by Beijing Separation Science \& Technology Development Co. Ltd. (Beijing, China). The optical signals were received and processed by an Agilent Chemstation through signal transformation with an Agilent 35900E A/D converter.

Chromatographic conditions: Several chiral columns with different chiral stationary phases including Lux cellulose1 (cellulose tris-(3,5-dimethylphenylcarbamate), CDMPC), Lux amylose-2 (amylose tris-(5-chloro-2-methylphenylcarbamate), ACMPC), Lux cellulose-2 (cellulose tris-(3chloro-4-methylphenylcarbamate), CCMPC), Lux cellulose-3 (cellulose tris-(4-methylbenzoate), CTMB) were used for enantioseparation on NP-HPLC and RP-HPLC. All chiral columns obtained from Phenomenex Incorporation (USA) were $250 \mathrm{~mm} \times 4.6 \mathrm{~mm}$, packed with $5 \mu \mathrm{m}$ particles. The chemical structures of these chiral stationary phases were shown in Fig. 2.

The mobile phases were different percentages of 2-propanol and hexane for NP-HPLC, acetonitrile and water for RP-HPLC. The column temperatures were varied from $5-40{ }^{\circ} \mathrm{C}$. In both

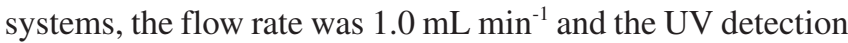
wavelength was set at $210 \mathrm{~nm}$. The injection volume was $10 \mu \mathrm{L}$.

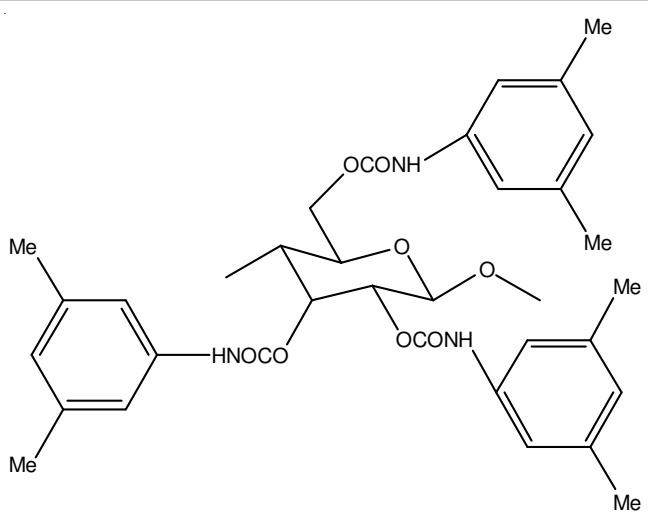

Lux cellulose-1, cellulose tris-(3,5-dimethylphenylcarbamate)

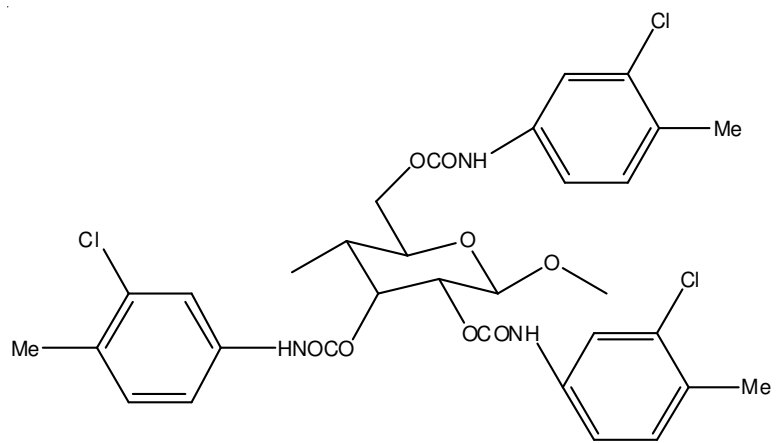

Lux cellulose-2, cellulose tris(3-chloro-4-methylphenylcarbamate)

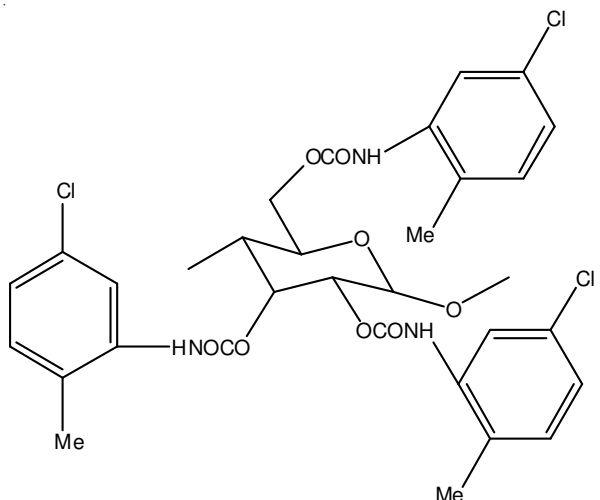

Lux amylose-2, amylose tris-(5-chloro-2-methyl-phenylcarbamate)

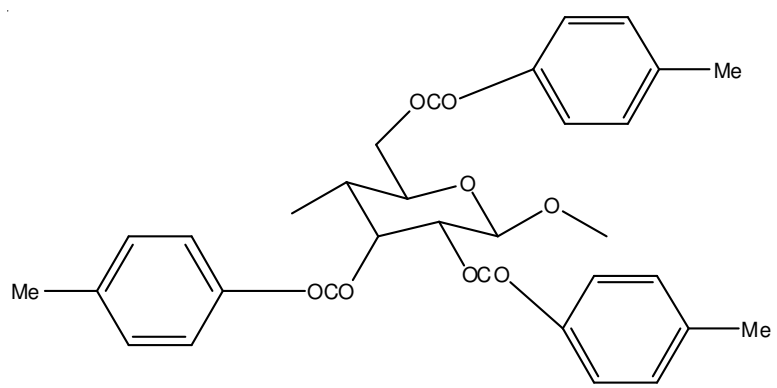

Lux cellulose-3, cellulose tris-(4-methylbenzoate)

Fig. 2. Chemical structures of four chiral stationary phases

The first-enantiomer capacity factor $\left(\mathrm{k}_{1}\right)$, the secondenantiomer capacity factor $\left(\mathrm{k}_{2}\right)$, the separation factor $(\alpha)$ and the resolutions (Rs) were calculated from the equations: $\mathrm{k}=$ $\left(\mathrm{t}-\mathrm{t}_{0}\right) / \mathrm{t}_{0}, \mathrm{t}_{0}$ was the void time at given conditions; $\alpha=\mathrm{k}_{2} / \mathrm{k}_{1} ; \mathrm{Rs}$ $=2\left(t_{2}-t_{1}\right) /\left(W_{1}+W_{2}\right), W_{1}$ and $W_{2}$ were the peak width of the first and second enantiomers, respectively. 


\section{RESULTS AND DISCUSSION}

Enantioseparation on NP-HPLC: The separation results on NP-HPLC were summarized in Table-1. Enantiomers of crufomate was completely separated (Rs $>1.5$ ) on Lux cellulose-1, Lux amylose-2 and Lux cellulose-2. With the decrease of 2-propanol in mobile phase, the Rs values of crufomate increased. The right $(+)$ rotation enantiomer was firstly eluted from Lux cellulose-1 and Lux amylose-2, but conversely the left (-) rotation enantiomer was firstly eluted from Lux cellulose-2 and Lux cellulose-3. Both enantiomers can't be eluted in 40 min from Lux cellulose- 2 when using 2-propanol/hexane (2/98, v/v) as mobile phase. Although crufomate enantiomers could not be significantly separated on Lux cellulose- 3 with UV detector, the elution orders could be given by optical rotation detector. The typical resolution chromatograms of crufomate were shown in Fig. 3.

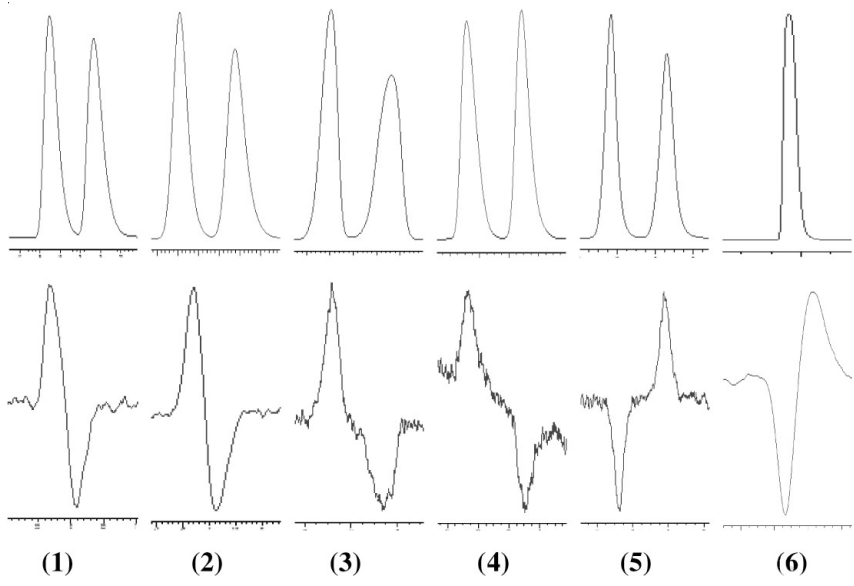

Fig. 3. Typical UV and OR chromatograms of crufomate enantiomers on Lux cellulose-1 (1) (3), Lux amylose-2 (2) (4) and Lux cellulose-2 (5), Lux cellulose-3 (6). Chromatographic conditions: ACN/water (40/60) with flow rate of $1.0 \mathrm{~mL} \mathrm{~min}{ }^{-1}$ at $20^{\circ} \mathrm{C}$ for (1) (2); 2-propanol/hexane (5/95) with flow rate of $1.0 \mathrm{~mL} \mathrm{~min}^{-1}$ at $20^{\circ} \mathrm{C}$ for (3) (4) (5); 2-propanol/ hexane $(1 / 99)$ with flow rate of $1.0 \mathrm{~mL} \mathrm{~min}^{-1}$ at $10{ }^{\circ} \mathrm{C}$ for $(6)$

Ellington ${ }^{7}$ had reported crufomate enantiomers can obtain separation on Chiralcel $^{\circledR} \mathrm{AD}$ with ethanol/heptane $(\mathrm{Rs}=2.8)$, on Chiralcel ${ }^{\circledR} \mathrm{OD}$ with ethanol/heptane $(\mathrm{Rs}=1.1)$ and on Chiralce ${ }^{\circledR} \mathrm{OJ}$ with ethanol/hexane $(\mathrm{Rs}=0.9)$. Chemically, the chiral stationary phase of Lux Cellulose-1 was CDMPC and same to that of Chiralcel ${ }^{\circledR}$ OD, Lux cellulose-2 was CCMPC and same to that of Chiralce ${ }^{\circledR} \mathrm{OZ}$, Lux cellulose-3 was CTMB and same to that of Chiralce ${ }^{\circledR} \mathrm{OJ}$, Lux amylose-2 was ACMPC and same to that of Chiralpak ${ }^{\circledR} \mathrm{AY}$, Chiralcel ${ }^{\circledR} \mathrm{AD}$ was ADMPC (amylose tris-(3,5-dimethylphenylcarbamate). In Ellington's report, the elution orders of crufomate enantiomers on ADMPC with ethanol/heptane and on CTMB with ethanol/hexane were $(-) /(+)$ and reverse to that on CDMPC with ethanol/heptane. But in this study, when the mobile phase was 2-propanol/ hexane, the elution orders on ACMPC were (+)/(-) and same to that on CDMPC. The reasons of reverse elution orders may be different solvent composition of ethanol/heptane and 2propanol/hexane, or different substituted groups on phenylcarbamate of ADMPC and ACMPC although they both are amylose-derivatized chiral stationary phases.

By comparison with present separation results, CCMPC showed better chiral discriminability for crufomate than other three chiral stationary phases. The substituted group of 3chloro-4-methyl on phenylcarbamate of cellulose-based chiral stationary phases was more effective on chiral discrimination than those of 3,5-dimethyl and 4-methyl and had stronger interaction with $(+)$-crufomate than (-)-crufomate. But this interaction was reverse on CDMPC and lead to preferential elution of (+)-enantiomer. Additionally, the change of organic modifiers from 2-propanol to ethanol didn't change the elution order on CTMB with (-)-enantiomer firstly eluted and the change from ethanol/heptane to 2-propanol/hexane also didn't change that on CDMPC.

In the enantiomer-chiral stationary phases interactions and recognition mechanisms, the key enantioselectivity was owed to derivatized glucose polymer, which interacted with enantiomers through hydrogen bonding, dipole moments, $\pi$ - $\pi$ interaction etc. ${ }^{13-15}$. Phenylcarbamate played significant roles not only in interacting with enantiomers directly, but also in keeping deep bonding with free hydroxyls of the glucose polymer ${ }^{16-18}$. By comparing the resolution results and elution orders in present study with Ellington's report, it could be suspected the polar modifier in the mobile phase may participate in the interaction between enantiomers and chiral stationary phases. The weaker polar solvent such as 2-propanol and lower concentration of the polar component caused better separation on NP-HPLC.

Enantioseparation on RP-HPLC: Acetonitrile was used as one component of mobile phase on RP-HPLC, for its low viscosity, low chemical reactivity, suitable eluting strength on

TABLE-1

ENANTIOMERIC SEPARATION OF CRUFOMATE ON NP-HPLC AT $20^{\circ} \mathrm{C}$

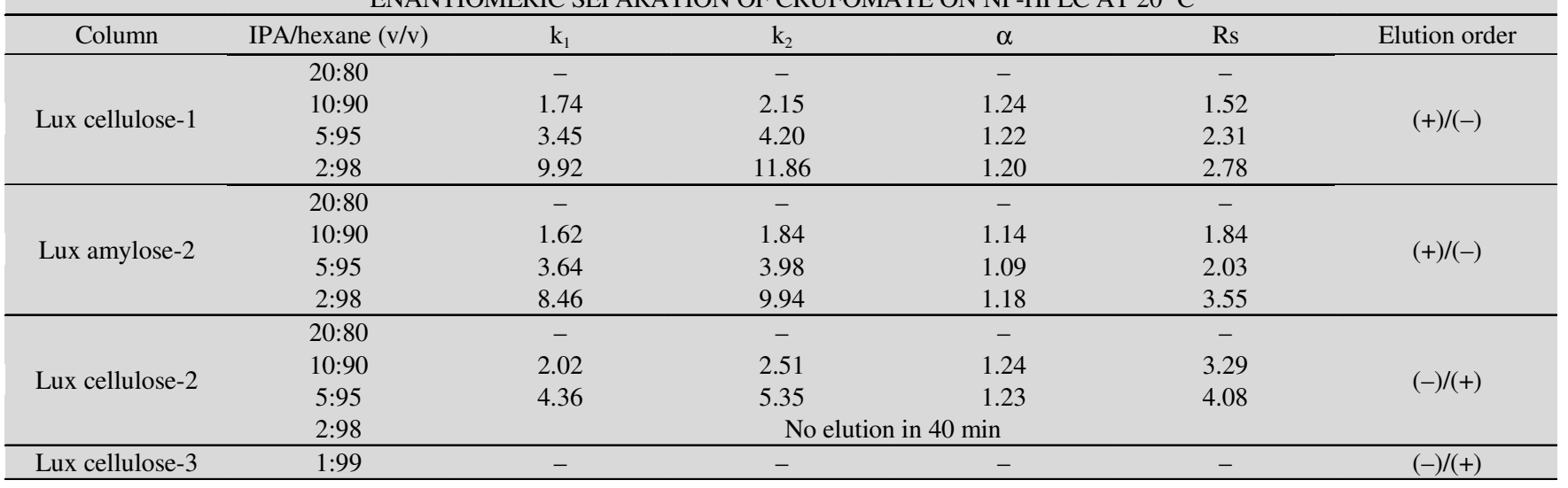


TABLE-2

ENANTIOMERIC SEPARATION OF CRUFOMATE ON RP-HPLC AT $20^{\circ} \mathrm{C}$

\begin{tabular}{|c|c|c|c|c|c|c|}
\hline Column & $\mathrm{CH}_{3} \mathrm{CN} /$ Water (v/v) & $\mathrm{k}_{1}$ & $\mathrm{k}_{2}$ & $\alpha$ & Rs & Elution order \\
\hline \multirow{5}{*}{ Lux cellulose-1 } & $30: 70$ & & & $\min$ & & \multirow{5}{*}{$(+) /(-)$} \\
\hline & $40: 60$ & 5.87 & 6.69 & 1.14 & 2.75 & \\
\hline & $50: 50$ & 2.24 & 2.52 & 1.13 & 2.09 & \\
\hline & 60:40 & 0.99 & 1.12 & 1.13 & 1.50 & \\
\hline & 70:30 & 0.51 & 0.58 & 1.13 & 1.13 & \\
\hline \multirow{5}{*}{ Lux amylose-2 } & $\begin{array}{l}30: 70 \\
\end{array}$ & 3.81 & 6.29 & 1.65 & 3.56 & \multirow{5}{*}{$(+) /(-)$} \\
\hline & $40: 60$ & 3.73 & 4.30 & 1.15 & 3.05 & \\
\hline & $50: 50$ & 1.62 & 1.80 & 1.11 & 1.92 & \\
\hline & 60:40 & 0.99 & 1.06 & 1.07 & 1.07 & \\
\hline & 70:30 & - & - & - & - & \\
\hline Lux cellulose- 2 & $30: 70$ & - & - & - & - & - \\
\hline Lux cellulose- 3 & $30: 70$ & - & - & - & - & - \\
\hline
\end{tabular}

the polysaccharide-type chiral stationary phases and outstanding ability to obtain good enantioselectivity ${ }^{19}$. The effects of volume proportion of acetonitrile on separation were investigated at $20{ }^{\circ} \mathrm{C}$. Table- 2 shows the chromatographic separation results on RP-HPLC. Baseline enantioseparation of crufomate on Lux cellulose-1, Lux amylose-2 was obtained on suitable conditions, while better resolution can't be achieved on Lux cellulose-2 and Lux cellulose-3. Rs values rose with the decrease of acetonitrile percentage in mobile phase. When percentage of acetonitrile/water was 70/30, Lux amylose-2 can't separate crufomate enantiomers but Lux cellulose-1 can partially separate them. Additionally, crufomate can't be eluted in $50 \mathrm{~min}$ from Lux cellulose-1 when using acetonitrile/water (30/70, v/v) as mobile phase.

The elution orders of crufomate on Lux cellulose- 1 and Lux amylose-2 on RP-HPLC were same to that on NP-HPLC with the (+)-enantiomer eluted firstly, indicated the change of mobile phase from 2-propanol/hexane on NP-HPLC to acetonitrile/water on RP-HPLC didn't affect stronger interactions of (-)-enantiomer with two chiral stationary phases than (+)enantiomer. However, this change significantly affected chiral discriminability of Lux cellulose-2 for crufomate and lead to noneffective separation on this column on RP-HPLC. Additionally, unlike detection of crufomate enantiomers on NPHPLC, their elution orders could not be obtained on Lux cellulose- 3 by both UV and optical rotation detectors after this change. The typical resolution chromatograms were shown in Fig. 3.

The hydrophobic combination, one of the enantiomerchiral stationary phases interactions, played a significant role to illustrate the enantioselectivity in aqueous/organic mobile phases, consisting of a buffer solution mixed with acetonitrile and/or methanol. In aqueous/organic mobile phases, all the strong and weak combinations were sensitive to the organic substance ${ }^{20,21}$. According to the enantioseparation on RPHPLC, it was apparently shown that the hydrophobic bondings of two enantiomers-chiral stationary phases were both weakened and the retention times were shorter with the increase of acetonitrile percentage in mobile phases. However, the resolutions values decreased because of different hydrophobic interactions of two enantiomers with chiral stationary phases.

As a novel separation method for crufomate enantiomers, RP-HPLC provided a faster reply compared with NP-HPLC for chiral stationary phases/mobile phase combination and could be used to develop more sensitive enantiomeric analytical method by combining with mass spectrometry. However, the resolutions can be also improved on NP-HPLC and would be helpful to obtain more adequate enantioseparation conditions to prepare optical pure enantiomers.

Influence of temperature on resolution: The effects of column temperatures from $5-40^{\circ} \mathrm{C}$ on NP-HPLC and RP-HPLC on different columns were investigated for enantioseparation. The results were summarized in Tables 3 and 4.

\section{TABLE-3}

IMPACT OF TEMPERATURE ON DIFFERENT COLUMNS ON NP-HPLC

\begin{tabular}{ccccccc}
\hline Column & $\begin{array}{c}\text { Temperature } \\
\left({ }^{\circ} \mathrm{C}\right)\end{array}$ & $\mathrm{k}_{1}$ & $\mathrm{k}_{2}$ & $\alpha$ & $\mathrm{Rs}$ & $\begin{array}{c}\text { Elution } \\
\text { order }\end{array}$ \\
\hline & 5 & 4.20 & 5.36 & 1.28 & 3.16 & \\
Lux & 10 & 3.91 & 4.98 & 1.27 & 3.07 & \\
cellulose- & 20 & 3.45 & 4.33 & 1.26 & 2.70 & $(+) /(-)$ \\
$1^{\mathrm{a}}$ & 30 & 3.11 & 3.80 & 1.22 & 2.25 & \\
& 40 & 2.82 & 3.37 & 1.20 & 1.88 & \\
\hline & 5 & 11.96 & 13.34 & 1.12 & 3.10 & \\
Lux & 10 & 11.45 & 12.78 & 1.12 & 3.18 & \\
amylose- & 20 & 10.70 & 11.94 & 1.12 & 3.50 & $(+) /(-)$ \\
$2^{\mathrm{b}}$ & 30 & 9.75 & 10.99 & 1.13 & 3.76 & \\
& 40 & 8.84 & 10.07 & 1.14 & 4.11 & \\
\hline & 5 & 4.97 & 6.27 & 1.26 & 4.23 & \\
Lux & 10 & 4.80 & 6.01 & 1.25 & 4.05 & \\
cellulose- & 20 & 4.53 & 5.56 & 1.23 & 4.13 & $(-) /+)$ \\
$2^{\mathrm{a}}$ & 30 & 4.37 & 5.29 & 1.21 & 4.00 & \\
& 40 & 4.34 & 5.16 & 1.19 & 3.81 & \\
\hline
\end{tabular}

${ }^{a}$ IPA/hexane (5/95) at flow rate of $1.0 \mathrm{~mL} \mathrm{~min}^{-1},{ }^{b} \mathrm{IPA} /$ hexane (2/98) at flow rate of $1.0 \mathrm{~mL} \mathrm{~min}^{-1}$.

\begin{tabular}{ccccccc}
\multicolumn{7}{c}{ TABLE-4 } \\
\multicolumn{7}{c}{$\begin{array}{l}\text { IMPACT OF TEMPERATURE ON } \\
\text { DIFFERENT COLUMNS ON RP-HPLC }\end{array}$} \\
\hline Column & $\begin{array}{c}\text { Temperature } \\
\left({ }^{\circ} \mathrm{C}\right)\end{array}$ & $\mathrm{k}_{1}$ & $\mathrm{k}_{2}$ & $\alpha$ & $\mathrm{Rs}$ & $\begin{array}{c}\text { Elution } \\
\text { order }\end{array}$ \\
\hline \multirow{4}{*}{ Lux } & 5 & 4.35 & 4.52 & 1.04 & 1.07 & \\
Amylose- & 10 & 4.04 & 4.25 & 1.05 & 1.38 & \\
$2^{\mathrm{a}}$ & 20 & 3.32 & 3.59 & 1.08 & 2.31 & $(+) /(-)$ \\
& 30 & 2.58 & 2.92 & 1.13 & 3.67 & \\
& 40 & 2.15 & 2.45 & 1.14 & 4.01 & \\
Lux & 5 & 7.08 & 8.12 & 1.15 & 2.75 & \\
Cellulose- & 10 & 6.68 & 7.63 & 1.14 & 2.71 & \\
$1^{\mathrm{a}}$ & 20 & 5.94 & 6.73 & 1.13 & 2.61 & $(+) /(-)$ \\
& 30 & 5.25 & 5.89 & 1.12 & 2.42 & \\
\hline${ }^{\mathrm{a}}$ ACN/Water(40/60) at flow rate of $1.0 \mathrm{~mL} \mathrm{~min}^{-1}$. & &
\end{tabular}

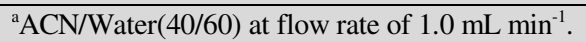


The van't Hoff equations as follows in chemical thermodynamics relates the change in temperature $(\mathrm{T})$ to the change in capacity factor given the standard enthalpy change $\left(\Delta \mathrm{H}^{\circ}\right)$ and entropy change $\left(\Delta S^{\circ}\right)$ for the process of analyte from the mobile phase to the stationary phase $\mathrm{e}^{22,23}$.

$$
\begin{gathered}
\ln \mathrm{k}=-\frac{\Delta \mathrm{H}^{\circ}}{\mathrm{RT}}+\frac{\Delta \mathrm{S}^{\circ}}{\mathrm{R}}+\ln \phi \\
\ln \alpha=-\frac{\Delta \Delta \mathrm{H}^{\circ}}{\mathrm{RT}}+\frac{\Delta \Delta \mathrm{S}^{\circ}}{\mathrm{R}}
\end{gathered}
$$

The second equation is derived from the first. $\Delta \Delta \mathrm{H}^{\mathrm{o}}$ and $\Delta \Delta \mathrm{S}^{\circ}$ are difference of $\Delta \mathrm{H}_{2}{ }^{\circ}-\Delta \mathrm{H}_{1}{ }^{\circ}$ and $\Delta \mathrm{S}_{2}{ }^{\circ}-\Delta \mathrm{S}_{1}{ }^{\circ}$, respectively. If plots of $\ln \mathrm{k}$ versus $1 / \mathrm{T}$ are linear, $-\Delta \mathrm{H}^{\circ} / \mathrm{R}$ and $\Delta \mathrm{S}^{\circ} / \mathrm{R}+\ln \mathrm{f}$ are the slope and intercept, respectively. For linear plots of $\ln \alpha$ versus $1 / \mathrm{T}$, the slope and intercept are $-\Delta \Delta \mathrm{H}^{\circ} / \mathrm{R}$ and $\Delta \Delta \mathrm{S} / \mathrm{R}$, respectively. The thermodynamic factors were listed in Table-5.

On NP-HPLC, the capacity factors, separation factors, resolution factors of crufomate enantiomers decreased with the increasing temperature on Lux cellulose-1 and Lux cellulose-2. However, the separation factors and resolution factors on Lux amylose- 2 were reverse to that. It was usual that the temperature was lower, the enantioselectivity of enantiomers was better on cellulose-based chiral stationary phases but worse on amylose-based chiral stationary phase $\mathrm{s}^{24,25}$. The resolution values increased from 3.10 at $5^{\circ} \mathrm{C}$ to 4.11 at $40{ }^{\circ} \mathrm{C}$ on above three columns. All plots of $\ln \mathrm{k}$ versus $1 / \mathrm{T}, \ln \alpha$ versus $1 / \mathrm{T}$ were good linearization. The values of $\Delta \mathrm{H}^{\circ}$ were negative on Lux cellulose-1, Lux cellulose-2 and Lux amylose-2. The $\Delta \mathrm{H}_{2}{ }^{\circ}$ values were more negative than the $\Delta \mathrm{H}_{1}{ }^{\circ}$ values on Lux cellulose- 1 and Lux cellulose- 2 , which meant the weaker interaction of first enantiomer with chiral stationary phases. On the contrary, the $\Delta \mathrm{H}_{2}{ }^{\circ}$ values were less negative than the $\Delta \mathrm{H}_{1}{ }^{\mathrm{o}}$ value on Lux amylose-2, suggested the stronger interaction of first enantiomer with chiral stationary phases. The $\Delta S^{\circ}$ values on Lux cellulose-1 were negative but they were positive on Lux cellulose-2. The $\Delta \mathrm{S}_{1}{ }^{\mathrm{o}}$ and $\Delta \mathrm{S}_{2}{ }^{\mathrm{o}}$ were negative and positive on Lux amylose-2, respectively. The value of $\Delta \Delta \mathrm{H}^{\circ}$ was positive on Lux amylose-2, suggested higher temperature was helpful to get better resolution. The values of $\Delta \Delta \mathrm{H}^{\circ}$ were negative on Lux cellulose- 1 and Lux cellulose-2, indicating lower temperature was helpful to get better resolution and reverse to that on Lux amylose-2. The $\Delta \Delta S^{\circ}$ values on Lux cellulose- 1 and Lux cellulose- 2 were negative, which meant that the enantioseparation on two columns were an enthalpy-driven progress. But the positive $\Delta \Delta S^{\circ}$ value on Lux amylose-2 was unmanageable which indicated that the enantioselectivity was not an enthalpydriven course.

On RP-HPLC, with the column temperature lower, the separation factors and resolution factors increased on Lux cellulose- 1 but decreased on Lux amylose-2. The separation factors on Lux amylose-2 were decreased from 1.14 at $40{ }^{\circ} \mathrm{C}$ to 1.04 at $5{ }^{\circ} \mathrm{C}$. The values of $\Delta \mathrm{H}^{\circ}$ were negative on Lux cellulose-1 and Lux amylose-2, indicating an enthalpy-driven progress. The $\Delta \mathrm{H}_{2}{ }^{\mathrm{o}}$ values were more positive than the $\Delta \mathrm{H}_{1}{ }^{\circ}$ values and the $\Delta \mathrm{S}^{\circ}$ values were negative both on Lux cellulose1 and Lux amylose-2. The value of $\Delta \Delta \mathrm{H}^{\circ}$ was negative on Lux cellulose-1, indicated lower temperature was constructive to obtain better separation and contrary to that on Lux amylose2 . The $\Delta \Delta S^{\circ}$ value on Lux amylose- 2 was positive, indicating not an enthalpy-driven progress.

\section{Conclusion}

In present study, crufomate enantiomers were separated on Lux cellulose-1, Lux amylose-2, Lux cellulose-2 and Lux cellulose- 3 on NP-HPLC and RP-HPLC. The effects of mobile phase percentage and column temperature on resolution were investigated. The elution orders on different chiral stationary phases were given by an optical rotation detector. In both HPLC systems, the baseline enantioseparations were obtained on Lux cellulose-1 and Lux amylose-2 and the elution orders were identical. The good resolution was achieved on Lux cellulose2 on NP-HPLC but difficult to get on RP-HPLC. The (-)-crufomate was firstly eluted on Lux cellulose- 2 on NP-HPLC, while the (+)-crufomate was firstly eluted on Lux cellulose-1 and Lux amylose-2 on NP-HPLC and RP-HPLC. Lower temperature was helpful to obtain better separation on cellulose-based chiral stationary phases, while higher temperature was more constructive on amylose-based chiral stationary phases both on NP-HPLC and RP-HPLC. Although baseline separation of crufomate enantiomers could not be obtained on Lux cellulose3 on NP-HPLC, the elution orders had been detected with the (-)-enantiomer firstly eluted. The substituted groups on cellulosebased chiral stationary phases significantly affect their chiral discriminabilities for crufomate and lead to reverse enantiomeric elution orders on different chiral stationary phases. This work is helpful to prepare enantiomer-pure crufomate for toxicological evaluation and develop effective analytical methods for study on enantioselective behaviours of crufomate in agriculture and environment.

TABLE-5

\begin{tabular}{|c|c|c|c|c|c|c|c|c|c|}
\hline System & Column & $\ln \mathrm{k}^{\mathrm{a}}=-\Delta \mathrm{H}^{\mathrm{o}} / \mathrm{RT}+\Delta \mathrm{S}^{\mathrm{o}} / \mathrm{R}$ & $\mathrm{R}_{1}^{2}$ & $\begin{array}{c}\Delta \mathrm{H}^{\circ} \\
(\mathrm{kJ} / \mathrm{mol})\end{array}$ & $\begin{array}{c}\Delta \mathrm{S}^{\mathrm{o}} \\
(\mathrm{J} / \mathrm{mol}) \\
\end{array}$ & $\ln \alpha=-\Delta \Delta \mathrm{H}^{\circ} / \mathrm{RT}+\Delta \Delta \mathrm{S}^{\circ} / \mathrm{R}$ & $\mathrm{R}_{2}^{2}$ & $\Delta \Delta \mathrm{H}^{\circ}$ & $\Delta \Delta \mathrm{S}^{\circ}$ \\
\hline \multirow{6}{*}{ NP-HPLC } & Lux & $\ln \mathrm{k}_{1}=990.8 / \mathrm{T}-2.133$ & 0.998 & -8.24 & -17.73 & \multirow{2}{*}{$\ln \alpha=163.1 / \mathrm{T}-0.336$} & \multirow[t]{2}{*}{0.958} & \multirow{2}{*}{-1.36} & \multirow[t]{2}{*}{-2.79} \\
\hline & Cellulose-1 & $\ln \mathrm{k}_{2}=1154 / \mathrm{T}-2.470$ & 1.000 & -9.59 & -20.54 & & & & \\
\hline & Lux & $\ln \mathrm{k}_{1}=738.9 / \mathrm{T}-0.167$ & 0.990 & -6.14 & -1.39 & \multirow{2}{*}{$\ln \alpha=-49.60 / T+0.284$} & \multirow{2}{*}{0.820} & \multirow{2}{*}{0.41} & \multirow{2}{*}{2.36} \\
\hline & Amylose-2 & $\ln \mathrm{k}_{2}=689.3 / \mathrm{T}+0.117$ & 0.994 & -5.73 & 0.97 & & & & \\
\hline & Lux & $\ln \mathrm{k}_{1}=349.4 / \mathrm{T}+0.334$ & 0.938 & -2.90 & 2.78 & \multirow{2}{*}{$\ln \alpha=145.5 / T-0.290$} & \multirow{2}{*}{0.998} & \multirow{2}{*}{-1.21} & \multirow{2}{*}{-2.41} \\
\hline & Cellulose-2 & $\ln \mathrm{k}_{2}=494.9 / \mathrm{T}+0.044$ & 0.970 & -4.11 & 0.37 & & & & \\
\hline \multirow{4}{*}{ RP-HPLC } & Lux & $\ln \mathrm{k}_{1}=1051 / \mathrm{T}-1.815$ & 0.997 & -8.74 & -15.09 & \multirow{2}{*}{$\ln \alpha=77.02 / \mathrm{T}-0.139$} & \multirow{2}{*}{0.993} & \multirow{2}{*}{-0.64} & \multirow{2}{*}{-1.16} \\
\hline & Cellulose-1 & $\ln \mathrm{k}_{2}=1128 / \mathrm{T}-1.954$ & 0.997 & -9.38 & -16.25 & & & & \\
\hline & Lux & $\ln \mathrm{k}_{1}=1551 / \mathrm{T}-4.042$ & 0.992 & -12.90 & -33.61 & \multirow{2}{*}{$\ln \alpha=-245.5 / T+0.92$} & \multirow{2}{*}{0.968} & \multirow{2}{*}{2.04} & \multirow{2}{*}{7.65} \\
\hline & Amylose-2 & $\ln \mathrm{k}_{2}=1796 / \mathrm{T}-4.962$ & 0.993 & -14.93 & -41.25 & & & & \\
\hline
\end{tabular}

VAN'T HOFF EQUATIONS AND THERMODYNAMIC PARAMETERS

${ }^{a} k_{1}$ and $k_{2}$ were respectively calculated for first- and second-eluted enantiomers of crufomate. 


\section{ACKNOWLEDGEMENTS}

This work was funded by National High Technology Research and Development Program of China (No. 2011AA100806).

\section{REFERENCES}

1. L.G. Costa, Clin. Chim. Acta, 366, 1 (2006).

2. B.K. Singh and A. Walker, FEMS Microbiol. Rev., 30, 428 (2006).

3. N. Kurihara and J. Miyamoto, Chirality in Agrochemicals, John Wiley \& Sons, Chichester (1998).

4. A.S. Garay, Nature, 271, 186 (1987)

5. A.W. Garrison, Green Chem., 6, 77 (2004).

6. J. Jarman, W. Jones, L. Howell and A.W. Garrison, J. Agric. Food Chem., 53, 6175 (2005)

7. J.J. Ellington, J.J. Evans and K. Prickett, J. Chromatogr. A, 928, 145 (2001).

8. Y. Wang, K. Tai and J. Yen, Ecotoxicol. Environ. Safety, 57, 346 (2004).

9. M.G. Nillos, J. Gan and D. Schlenk, J. Chromatogr. B, 878, 1277 (2010).

10. L. Li, S.S. Zhou, L.X. Jin, C. Zhang and W.P. Li, J. Chromatogr. B, 878, 1264 (2010).

11. Y.H. Zhu, Z.R. Wang and B.Q. Li, Encyclopedia of Agricultural Chemistry (2006).
12. P. Schmitt, A.W. Garrison, D. Freitag and A. Kettrup, J. Chromatogr. A, 792, 419 (1997).

13. E. Yashima, J. Chromatogr. A, 906, 105 (2001).

14. Y. Okamoto and E. Yashima, Angew. Chem. Int. Ed., 37, 1020 (1998).

15. P. Wang, S.R. Jiang and Z.Q. Zhou, J. Agric. Food Chem., 54, 1577 (2006).

16. E.N. Shapovalova, O.A. Shpigun, L.M. Nesterova and M.Y. Belov, J. Anal. Chem., 59, 255 (2004).

17. Y. Zhou, L. Li, K.D. Lin, X.P. Zhu and W.P. Liu, Chirality, 21, 421 (2009).

18. J. Qiu, S.H. Dai, S.M. Yang, T.T. Chai and M. Bie, Chirality, 23, 479 (2011)

19. T. Zhang, D. Nguyen and P. Franco, J. Chromatogr. A, 1217, 1048 (2010).

20. A. Berthod, Anal. Chem., 78, 2093 (2006).

21. L.M. Peng, S. Jayapalan, B. Chankvetadze and T. Farkas, J. Chromatogr. A, 1217, 6942 (2010)

22. R.J. Smith, D.R. Taylor and S.M. Wilkins, J. Chromatogr. A, 697, 591 (1995).

23. A. Péter, E. Vékes and D.W. Armstrong, J. Chromatogr. A, 958, 89 (2002).

24. O. Gyllenhaal and M. Stefansson, Chirality, 17, 257 (2005).

25. T. O'Brien, L. Crocker, R. Thompson, K. Thompsom, P. Toma, D. Conlon, B. Feibush, C. Moeder, G. Bicker and N. Grinberg, Anal. Chem., 69, 1999 (1997). 